\title{
TERMINAL QUOTIENT SINGULARITIES IN DIMENSIONS THREE AND FOUR
}

\author{
DAVID R. MORRISON ${ }^{1}$ AND GLENN STEVENS ${ }^{2}$
}

\begin{abstract}
We classify isolated terminal cyclic quotient singularities in dimension three, and isolated Gorenstein terminal cyclic quotient singularities in dimension four. In addition, we give a new proof of a combinatorial lemma of G. K. White using Bernoulli functions.
\end{abstract}

Let $X$ be a smooth algebraic variety over $\mathbf{C}$, and let $\omega_{X}$ be the canonical bundle of $X$. For each $n>0$, if $\Gamma\left(X, \omega_{X}^{\otimes n}\right) \neq \varnothing$, there is a natural pluricanonical map $\phi_{n}$ : $X \rightarrow \mathbf{P} \Gamma\left(X, \omega_{X}^{\otimes n}\right)^{*}$. An algebraic variety is of general type if $\phi_{n}$ is a birational map for $n$ sufficiently large. For a variety of general type, the pluricanonical images $\phi_{n}(X)$ are the most natural birational models of $X$ to study.

Canonical singularities are the singularities which may occur in the pluricanonical models of varieties of general type. In dimension 1, the pluricanonical models are smooth so there are no canonical singularities; in dimension 2 the canonical singularities coincide with the classical rational double points. One characterization of the rational double points is as quotient singularities: if $G$ is any finite subgroup of $\mathrm{Sl}(2, \mathbf{C})$, then the quotient $\mathrm{C}^{2} / G$ has a rational double point, and every rational double point is analytically isomorphic to such a quotient singularity. Reid and Shepherd-Barron [10], and independently Tai [14], have given a condition for quotient singularities to be canonical in arbitrary dimensions (although not all canonical singularities are quotient singularities in dimensions greater than two).

Terminal singularities are a class of canonical singularities which play an important role in birational geometry (as evidenced by recent work of Mori [8], Reid [12], and Tsunoda [15]). In this note we study cyclic quotient singularities which are terminal. In dimension three we explicitly describe all isolated terminal cyclic quotient singularities, while in dimension four, we describe isolated terminal cyclic quotient singularities which are also Gorenstein. The description uses a combinatorial lemma due to G. K. White [17]; we have given a new proof of this lemma (Corollary 1.4 below) using Bernoulli functions.

Received by the editors March 31, 1983.

1980 Mathematics Subject Classification. Primary 14B05; Secondary 10A40, 14L30, 32B30, 52A25.

Key words and phrases. Bernoulli functions, canonical singularity, Gorenstein ring, quotient singularity, terminal singularity.

${ }^{1}$ National Science Foundation Postdoctoral Fellow.

${ }^{2}$ Partially supported by National Science Foundation Grant MCS 82-01762. 
1. Bernoulli functions. The material in this section is all quite standard (except for the last two corollaries); we have adapted the presentation in the first chapter of Kubert and Lang [7] to suit our purposes.

If $x$ is a rational number, we let $\langle x\rangle$ denote the rational number such that $x \equiv\langle x\rangle \bmod \mathbf{Z}$, and $0 \leqslant\langle x\rangle<1$. Define

$$
\mathbf{B}_{1}(x)= \begin{cases}\langle x\rangle-\frac{1}{2} & \text { if } x \text { is not an integer, } \\ 0 & \text { if } x \text { is an integer. }\end{cases}
$$

Let $N$ be a natural number, let $C(N)=(1 / N) \mathbf{Z} / \mathbf{Z}$, and let $G(N)=(\mathbf{Z} / N \mathbf{Z})$. $G(N)$ acts on $C(N)$ by multiplication, and there is an embedding $G(N) \rightarrow C(N)$ given by $a \rightarrow a / N$.

Let $\chi: G(N) \rightarrow \mathrm{C}^{*}$ be a character of conductor $N$ and define

$$
\mathbf{B}_{1, \chi}=\sum_{a \in G(N)} \chi(a) \mathbf{B}_{1}\left(\frac{a}{N}\right) .
$$

The following classical theorem is essentially due to Dirichlet; a nice proof can be found in [5, §2, Theorem 2].

THEOREM 1.1. If $\chi$ is an odd character (that is, $\chi(-a)=-\chi(a)$ for all $a \in G(N)$, where $N$ is the conductor of $\chi)$, then $B_{1, \chi} \neq 0$.

Now fix a natural number $N>2$, and denote $C(N)$ and $G(N)$ by $C$ and $G$, respectively. Let $V=\mathbf{C}(G)$ be the group algebra of $G$ generated (as a C-vector space) by elements $\sigma_{a}$ for $a \in G$. We define a function $S$ : $C \rightarrow V$ by

$$
S(x)=\sum_{a \in G} \mathbf{B}_{1}(a x) \sigma_{a} .
$$

Let $W$ be the subspace of $V$ generated by $\{S(x): x \in C\}$, and let $\Lambda=\operatorname{Ann}(W) \subset V^{*}$. For each $a \in G$, let $\lambda_{a}=\sigma_{a}^{*}+\sigma_{-a}^{*} \in V^{*}$. Note that

$$
\lambda_{a}(S(x))=\mathbf{B}_{1}(a x)+\mathbf{B}_{1}(-a x)=0
$$

for all $x$ since $\mathbf{B}_{1}$ is an odd function. Thus, $\lambda_{a} \in \Lambda$.

Proposition 1.2. $\Lambda$ is generated (as a C-vector space) by $\left\{\lambda_{a}: a \in G\right\}$. In particular, $\operatorname{dim}_{\mathbf{C}}(\Lambda)=\operatorname{dim}_{\mathbf{C}}(W)=\phi(N) / 2$, where $\phi$ is Euler's $\phi$-function.

Proof. Let $\chi$ be an (arbitrary) odd character on $G, M$ the conductor of $\chi$, and $H=G(M)$. There is a well-defined map $H \rightarrow C=(1 / N) \mathbf{Z} / \mathbf{Z}$ given by $a \rightarrow a / M$. We define an element $w_{\chi} \in W$ by

$$
w_{\chi}=\sum_{a \in H} \chi(a) S\left(\frac{a}{M}\right) .
$$

In terms of the given basis of $V$, we may write

$$
w_{\chi}=\sum_{b \in G}\left(\sum_{a \in H} \chi(a) \mathbf{B}_{1}\left(\frac{a b}{M}\right)\right) \sigma_{b} .
$$


Thus, the coefficient of $\sigma_{1}$ is

$$
\sum_{a \in H} \chi(a) \mathbf{B}_{1}\left(\frac{a}{M}\right)=\mathbf{B}_{1, \chi} \neq 0
$$

so $w_{x} \neq 0$.

There is a natural action of $G$ on $V=\mathbf{C}(G)$. Under this action, $c \in G$ sends $w_{\chi}$ to

$$
\begin{aligned}
\sum_{b \in G}\left(\sum_{a \in H} \chi(a) \mathbf{B}_{1}\left(\frac{a b}{M}\right)\right) \sigma_{b} & =\sum_{b^{\prime} \in G} \sum_{a \in H} \chi(a) \mathbf{B}_{1}\left(\frac{a c^{-1} b^{\prime}}{M}\right) \sigma_{b^{\prime}} \\
& =\sum_{b^{\prime} \in G} \sum_{a^{\prime} \in H} \chi\left(a^{\prime} c\right) \mathbf{B}_{1}\left(\frac{a^{\prime} b^{\prime}}{M}\right) \sigma_{b^{\prime}}=\chi(c) w_{\chi} .
\end{aligned}
$$

Thus, $w_{\chi}$ lies in the $\chi$-eigenspace of the $G$-action. Since each $w_{\chi} \neq 0$, we see that $\left\{w_{\chi}: \chi\right.$ is an odd character $\}$ is a linearly independent subset of $W$, so $\operatorname{dim}_{\mathbf{C}}(W) \geqslant$ $\phi(N) / 2$. On the other hand, by considering the elements $\lambda_{a}$, we see that $\operatorname{codim}_{\mathbf{C}}(W)$ $=\operatorname{dim}_{\mathbf{C}}(\Lambda) \geqslant \phi(N) / 2$. Since $\operatorname{dim}_{\mathbf{C}}(V)=\phi(N)$, the inequalities are equalities, and the proposition follows.

Corollary 1.3. Let $a, b, c, d \in G$ and suppose, for all $x \in C, \mathbf{B}_{1}(a x)+\mathbf{B}_{1}(b x)+$ $\mathbf{B}_{1}(c x)=\mathbf{B}_{1}(d x)$. Then, after reordering $a, b, c$, we have $a \equiv-b(N)$, and $c \equiv d(N)$.

Proof. Let $\lambda=\sigma_{a}^{*}+\sigma_{b}^{*}+\sigma_{c}^{*}-\sigma_{d}^{*} \in V^{*}$. By hypothesis, $\lambda \in \Lambda$. But $\Lambda$ is generated by $\left\{\sigma_{a}^{*}+\sigma_{-a}^{*}\right\}$, so the corollary is clear.

Corollary 1.4 (White [17, Theorem 2], Frumkin [3] And Danilov [2]). Let $a$, $b, c$ be integers relatively prime to $N$. Suppose, for all $k \in \mathbf{Z}-N \mathbf{Z},\langle a k / N\rangle+$ $\langle b k / N\rangle+\langle c k / N\rangle\rangle$ 1. Then, after reordering $a, b, c$, we have $a+b \equiv 0(N)$.

Proof. First note that if $\langle a k / N\rangle+\langle b k / N\rangle+\langle c k / N\rangle \geqslant 2$, then

$\left\langle-\frac{a k}{N}\right\rangle+\left\langle-\frac{b k}{N}\right\rangle+\left\langle-\frac{c k}{N}\right\rangle=1-\left\langle\frac{a k}{N}\right\rangle+1-\left\langle\frac{b k}{N}\right\rangle+1-\left\langle\frac{c k}{N}\right\rangle \leqslant 3-2=1$, which contradicts our hypothesis. Thus,

$$
1<\left\langle\frac{a k}{N}\right\rangle+\left\langle\frac{b k}{N}\right\rangle+\left\langle\frac{c k}{N}\right\rangle<2
$$

Let $d=a+b+c$. Since

$$
\left\langle\frac{a k}{N}\right\rangle+\left\langle\frac{b k}{N}\right\rangle+\left\langle\frac{c k}{N}\right\rangle \equiv\left\langle\frac{d k}{N}\right\rangle \bmod \mathbf{Z},
$$

we must have

$$
\left\langle\frac{a k}{N}\right\rangle+\left\langle\frac{b k}{N}\right\rangle+\left\langle\frac{c k}{N}\right\rangle=1+\left\langle\frac{d k}{N}\right\rangle>1
$$

for each $k \in \mathbf{Z}-N \mathbf{Z}$. In particular, $d$ is relatively prime to $N$. In addition, by subtracting $\frac{3}{2}$ from both sides of the above equation, we get

$$
\mathbf{B}_{1}(a x)+\mathbf{B}_{1}(b x)+\mathbf{B}_{1}(c x)=\mathbf{B}_{1}(d x)
$$

for all $x=k / N \notin \mathbf{Z}$. On the other hand, this identity is trivially satisfied for $x=0$. This corollary now follows from the preceding one. 
2. Quotient singularities. Throughout this section $\Gamma$ will be a finite subgroup of $\mathrm{Gl}(n, \mathbf{C})$, and $X_{\Gamma}=\mathbf{C}^{n} / \Gamma$. We will study the singularity of $X_{\Gamma}$ at the origin $O \in \mathbf{C}^{n}$.

$g \in \Gamma$ is called a quasi-reflection if $\operatorname{rank}(g-I)=1$. A classical theorem of Shephard and Todd [13] and Chevalley [1] says that $X_{\Gamma}$ is smooth if and only if $\Gamma$ is generated by quasi-reflections. If $\Gamma$ is arbitrary, let $\Gamma_{q}$ be the largest subgroup of $\Gamma$ generated by quasi-reflections, and let $\tilde{\Gamma}=\Gamma / \Gamma_{q}$. Then it is clear that $X_{\Gamma} \cong X_{\tilde{\Gamma}}$; in studying quotient singularities, we may therefore restrict to groups $\Gamma$ such that $\Gamma_{q}=\{I\}$. Such a group is called small.

The following lemma is quite standard (cf. [4 or 9] for a proof).

Lemma 2.1. Let $\Gamma \subset \mathrm{Gl}(n, \mathbf{C})$ be a small group, and let $S=\left\{x \in \mathbf{C}^{n} \mid g(x)=x\right.$ for some $g \neq 1\}$. Then the singular locus of $X_{\Gamma}$ is $S / \Gamma$.

COROllaRY 2.2. If $\Gamma \subset \mathrm{Gl}(n, \mathrm{C})$ is a small cyclic group of order $N$, then $X_{\Gamma}$ has an isolated singularity if and only if all eigenvalues of a generator $g$ of $\Gamma$ are primitive Nth roots of unity.

The duality sheaf $\omega_{X_{\Gamma}}$ of a quotient singularity has been studied by Khinich [6], Watanabe [16], Reid and Shephard-Barron [10], Reid [11], and Tai [14]. We summarize their results as follows.

THEOREM 2.3. Let $\Gamma \subset \operatorname{Gl}(n, \mathbf{C})$ be a small group. For each $g \in \Gamma$ of order $M \neq 1$ and each primitive Mth root of unity $\zeta$, write the eigenvalues of $g$ as $\zeta^{a_{1}}, \ldots, \zeta^{a_{n}}$, with $0 \leqslant a_{i}<M$, and define $e(g, \zeta)=a_{1} / M+\cdots+a_{n} / M$.

(i) (Khinich and Watanabe) $X_{\Gamma}$ is Gorenstein if and only if $\Gamma \subset \operatorname{Sl}(n, \mathbf{C})$.

(ii) (Reid, Shephard-Barron, and Tai) $X_{\Gamma}$ is canonical if and only if $e(g, \zeta) \geqslant 1$ for all primitive $\zeta$ and all $g \neq 1$.

(iii) (Reid) $X_{\Gamma}$ is terminal if and only if $e(g, \zeta)>1$ for all primitive $\zeta$ and all $g \neq 1$.

We can now state our main result.

THEOREM 2.4. Let $\Gamma \subset \mathrm{Gl}(n, \mathrm{C})$ be a small cyclic group of order $N$.

(i) If $n=3$, then $X_{\Gamma}$ is an isolated terminal singularity if and only if, for each $g \in \Gamma$, $\operatorname{det} g$ is one of the eigenvalues of $g$.

(ii) If $n=4$, then $X_{\Gamma}$ is an isolated Gorenstein terminal singularity if and only if $\Gamma \subset \mathrm{Sl}(2, \mathrm{C}) \times \mathrm{Sl}(2, \mathrm{C}) \subset \mathrm{Gl}(4, \mathrm{C})$ in such a way that the projections $\operatorname{pr}_{i}: \Gamma \rightarrow \operatorname{Sl}(2, \mathrm{C})$ are faithful representations of $\Gamma$.

Remark 2.5. Choose a primitive $N$ th root of unity $\zeta$. In suitable coordinates on $\mathrm{C}^{n}$, the conditions above are equivalent to

(i) There is some $a$ with $(a, N)=1$ such that $\Gamma$ is generated by

$$
\left[\begin{array}{lll}
\zeta & & \\
& \zeta^{a} & \\
& & \zeta^{-a}
\end{array}\right]
$$


(ii) There is some $a$ with $(a, N)=1$ such that $\Gamma$ is generated by

$$
\left[\begin{array}{llll}
\zeta & & & \\
& \zeta^{-1} & & \\
& & \zeta^{a} & \\
& & & \zeta^{-a}
\end{array}\right]
$$

This provides the explicit description promised in the introduciton.

Proof. (i) Let $g$ be a generator of $\Gamma, \zeta$ a primitive $N$ th root of unity, and $\zeta^{a}, \zeta^{b}, \zeta^{c}$ the eigenvalues of $g$, with $0 \leqslant a, b, c<N$. By Corollary $1.2 X_{\Gamma}$ is isolated if and only if $(a, N)=(b, N)=(c, N)=1$. On the other hand, by 2.3(iii), $X_{\Gamma}$ is terminal if and only if $\langle a k / N\rangle+\langle b k / N\rangle+\langle c k / N\rangle>1$ for each $k \in \mathbf{Z}-N \mathbf{Z}$. By Corollary 1.4 we have $a+b \equiv 0(N)$ after rearranging, so the determinant is $\zeta^{c}$, which is one of the eigenvalues.

(ii) Let $g$ be a generator of $\Gamma, \zeta$ a primitive $N$ th root of unity, and $\zeta^{a}, \zeta^{b}, \zeta^{c}, \zeta^{d}$ the eigenvalues of $g$ with $0 \leqslant a, b, c, d<N$. By Corollary 1.2 and Theorem 2.3(i) and (iii), $X_{\Gamma}$ is isolated terminal Gorenstein if and only if $a+b+c+d \equiv 0(N)$, $(a, N)=(b, N)=(c, N)=(d, N)=1$, and

$$
\left\langle\frac{a k}{N}\right\rangle+\left\langle\frac{b k}{N}\right\rangle+\left\langle\frac{c k}{N}\right\rangle+\left\langle\frac{d k}{N}\right\rangle>1
$$

for all $k \in \mathbf{Z}-N \mathbf{Z}$. Since $a+b+c+d \equiv 0(N)$, we see that

$$
\left\langle\frac{a k}{N}\right\rangle+\left\langle\frac{b k}{N}\right\rangle+\left\langle\frac{c k}{N}\right\rangle+\left\langle\frac{d k}{N}\right\rangle
$$

is an integer, so that this is true if and only if

$$
\left\langle\frac{a k}{N}\right\rangle+\left\langle\frac{b k}{N}\right\rangle+\left\langle\frac{c k}{N}\right\rangle+\left\langle\frac{d k}{N}\right\rangle \geqslant 2
$$

or

$$
\left\langle\frac{a k}{N}\right\rangle+\left\langle\frac{b k}{N}\right\rangle+\left\langle\frac{c k}{N}\right\rangle \geqslant 2-\left\langle\frac{d k}{N}\right\rangle=1+\left\langle-\frac{d k}{N}\right\rangle .
$$

This is greater than 1 , so we may again apply Corollary 1.4 , to get $a+b \equiv 0(N)$ after rearranging; hence, $c+d \equiv 0(N)$ as well, and we see that $\Gamma \subset \operatorname{Si}(2, \mathrm{C}) \times$ $\operatorname{Sl}(2, \mathrm{C})$ with each induced representation in $\operatorname{Sl}(2, \mathrm{C})$ being faithful.

\section{REFERENCES}

1. C. Chevalley, Invariants of finite groups generated by reflections, Amer. J. Math. 77 (1955), 778-782.

2. V. I. Danilov, The birational geometry of toric threefolds, Izv. Akad. Nauk SSSR Ser. Mat. 46 (1982), 972-981; English transl., Math. USSR-Izv. 16 (1982).

3. M. A. Frumkin, Description of elementary three-dimensional polyhedra, First All-Union Conference on Statistical and Discrete Analysis of Non-Numerical Information, Experimental Bounds and Discrete Optimization, Abstract of Conference Reports, Moscow-Alma-Ata, 1981. (Russian)

4. A. Fujiki, On resolutions of cyclic quotient singularities, Publ. Res. Inst. Math. Sci. 10 (1974), 293-328.

5. K. Iwasawa, Lectures on p-adic L-functions, Princeton Univ. Press, Princeton, N.J., 1972.

6. V. A. Khinich, On the Gorenstein property of the ring of invariants of a Gorenstein ring, Izv. Akad. Nauk SSSR Ser. Mat. 40 (1976), 50-56; English transl., Math. USSR-Izv. 10 (1976), 47-53.

7. D. S. Kubert and S. Lang, Modular units, Springer-Verlag, Berlin and New York, 1981. 
8. S. Mori, Threefolds whose canonical bundles are not numerically effective, Ann. of Math. (2) 116 (1982), 133-176.

9. D. Prill, Local classification of quotients of complex manifolds by discontinuous groups, Duke Math. J. 34 (1967), 375-386.

10. M. Reid, Canonical 3-folds, Géometrie Algebrique d'Angers (A. Beauville, ed.), Sijthoof and Noordhoff: Alphen aan den Rijn, 1980, pp. 273-310.

11. Minimal models of canonical 3-folds, Algebriac Varieties and Analytic Varieties (S. Iitaka, ed.), Advanced Studies in Pure Math., vol. 1, North-Holland, Amsterdam, 1983.

12. __ Decomposition of toric morphisms, Arithmetic and Geometry: Papers Dedicated to I. R. Shafarevich (M. Artin and J. Tate, eds.), Birkhäuser, Boston, Mass., 1983.

13. G. C. Shephard and J. A. Todd, Finite unitary reflection groups, Canad. J. Math. 6 (1954), 274-304.

14. Y. S. Tai, On the Kodaira dimension of the moduli space of Abelian varieties, Invent. Math. 68 (1982), 425-439.

15. S. Tsunoda, Degeneration of minimal surfaces with non-negative Kodaira dimension, Proc. Sympos. Algebraic Geometry, Kinosaki, Japan, 1981. (Japanese)

16. K. Watanabe, Certain invariant subrings are Gorenstein. I, II, Osaka J. Math. 11 (1974), 1-8, 379-388.

17. G. K. White, Lattice tetrahedra, Canad. J. Math. 16 (1964), 389-397.

Department of Mathematics, Princeton University, Princeton, New Jersey 08544

Department of Mathematics, Rutgers University, New Brunswick, New Jersey 08903 